\title{
28 Research Square \\ Identification of miR-338-5p and miR-4776-3p as mediators of articular cartilage degeneration
}

\section{Jiaxu Wang}

Strategic Support Froce Medical Center

\section{Xin Guan}

Strategic Support Force Medical Center

Lele Zhang

Strategic Support Force Medical Center

Shuilin Shao

Strategic Support Force Medical Center

\section{Bo Gao}

Strategic Support Force Medical Center

Haixia Li

Strategic Support Force Medical Center

Litao Huo

Strategic Support Force Medical Center

Shibo Huang

Strategic Support Force Medical Center

Jigong Wu ( 1376576929@qq.com)

Strategic support force medical center

\section{Xiang Cui}

General Hospital of Chinese PLA

\section{Research Article}

Keywords: miR-338-5p, miR-4776-3p, cartilage, osteoarthritis

Posted Date: September 21st, 2021

DOl: https://doi.org/10.21203/rs.3.rs-915563/v1

License: (9) This work is licensed under a Creative Commons Attribution 4.0 International License. Read Full License 


\section{Abstract \\ Purpose}

Osteoarthritis is one of the most common chronic diseases nowadays, and can cause serious physical illness and economic burden. Cartilage degeneration is one of primary reasons for osteoarthritis, but there were not thorough studies on cartilage lesions.

\section{Methods}

miR-338-5p and miR-4776-3p were overexpressed and knocked down in SW1353 cells. Dual luciferase assay is used to detect the binding of miR-338-5p, miR-4776-3p and SOX6. The expression of MMP13, TNFA, IL6, MCP1, PARP, RunX2, ALP, OPN, WNT4, WNT16, TRACP and CTSK was quantified by qPCR. And the expression of WNT4 at the protein level was quantified by WB.

\section{Results}

In this study, we found miR-338-5p and miR-4776-3p showed significantly high expression on cartilage in osteoarthritis through bioinformatics methods, and verified miR-338-5p and miR-4776-3p can improve catabolism, and trigger biological processes such as inflammation and apoptosis. Meanwhile we validated miR-338-5p and miR-4776-3p may play negative regulation in the process of bone biology.

\section{Conclusions}

Our study showed that miR-338-5p and miR-4776-3p can be regarded as medium of articular cartilage degeneration, and it provided new biomarker of early diagnosis in cartilage disease and osteoarthritis.

\section{Introduction}

With growth of aging people, osteoarthritis is increasingly become main source of pain, disability and socioeconomic consumption worldwide [1]. During osteoarthritis period, cartilage tissue in the joints will corrode, thereby causing pain and serious loss of patients' joint function [2]. Cartilage degeneration is one of main reasons in osteoarthritis, but we have not fully understood the cellular and molecular mechanisms of its occurrence and development [3]. At present, there is no better method which can treat osteoarthritis caused by cartilage degeneration. Therefor disease prevention and early diagnosis appear to be necessary.

Micro-RNA (miRNA) is a kind of endogenous, small RNA with a length of about 20-24 nucleotide, and it has multiple important regulation role in cells. It enables to target most protein-coding transcripts, nearly participating in animals' various development and pathological processes, such as cell proliferation, 
migration, apoptosis and autophagy $[4,5]$. miRNA expression also causes many diseases, such as osteoporosis, diabetes, obesity and cancers [6-9], while some miRNA directly participate in inflammatory reaction and apoptosis process [10-13], which provides good reference for studies on osteoarthritis. Recent studies have showed that miR-140 played an important role in cartilage degeneration [14], however, similar studies on miRNA are rarely seen.

In this study, we tried to explore and verify new miRNA which plays an important role in cartilage degeneration. First, we downloaded 2,100 miRNA expression data of patients with lumbar disc herniation (LDH) and patients with osteoarthritis of spine (FJOA) from NCBI GEO database, and made bioinformatics analysis, which found miR-4776-3p and miR-338-5p showed significantly high expression in FJOA. Then, we explored functions of miR-4776-3p and miR-338-5p by utilizing miRNA mimics and miRNA inhibitors, which obtained these two miRNAs participated in inflammatory reaction, catabolism and apoptosis process. Finally, we predicted target genes of miR-4776-3p and miR-338-5p via Target scan website, and verified target binding of miR-4776-3p with SOX2 and miR-338-5p to SOX9 through experiments. This study, through bioinformatics analysis and experimental verification, proved initially the role of miR-4776-3p and miR-338-5p in cartilage degeneration, providing new target and treatment regimen for early diagnosis of osteoarthritis.

\section{Materials And Methods}

\section{Data download}

At initial stage, we searched miRNA expression database related to cartilage in GEO database of NCBI (https://www.ncbi.nlm.nih.gov/geo/). We only selected samples of patients with cartilage disease and healthy persons, and ruled out databases derived from animals and human cells, finally only GSE79258 was found to conform to our requirements and used as subsequent analysis and verification. GSE79258 is GPL21599 (miRCURY LNA micro-RNA Array, 7th generation, miRBase 20) sequencing platform based data, including 2 cartilage samples from LDH patients and 2 cartilage samples from FJOA patients.

\section{Identification of differently expressed miRNA (DE-miRNA)}

We made differential analysis for data downloaded by using "Limma" package in R language, so as to obtain differently expressed miRNA in LDH and FJOA. We set $|\log 2 \mathrm{FC}|>1$ and $\mathrm{P} \otimes 0.05$ as threshold of identifying DE-miRNA. Heat Map was created by "pheatmap" package in R language, and Volcano Plot was created by Graph Pad Prism 7.

\section{Prediction of potential transcription factors}

The upstream transcription factors of DE-miRNAs were predicted by FunRich software, which is a standalone software tool mainly used for functional enrichment and interaction network analysis of genes and proteins. We DE-miRNAs to obtain their upstream transcription factors, and present the top ten transcription factors according to $P$ value. 


\section{GO annotation and KEGG pathway enrichment analysis}

The GO functional annotation and KEGG pathway enrichment analysis for the DE-genes were conducted by Enrichr, which is a comprehensive resource for curated miRNA sets and a search engine that accumulates biological knowledge for further biological discoveries

(http://amp.pharm.mssm.edu/Enrichr/). We input DE-miRNAs, and conducted biological process (BP), cellular component (CC), and molecular function (MF) functional annotation and KEGG pathway enrichment analysis. The top ten results are presented according to $P$ value, with $P<0.05$ considered as statistically significant.

\section{Predication of potential target gene of DE-miRNA}

We predicated downstream target gene of DE-miRNA by using TargetScanHuman (http://www.targetscan.org/vert_72/). TargetScanHuman is a website which predicts miRNA target gene, including miRNA target gene results of 10 species such as human, mouse, rats, opossum, chimpanzees, rhesus monkeys, bovine, dogs, chicken and frog, and it can clearly see binding of miRNA and target gene. TargetScan predicted biological target of miRNA by searching conserved 8mer, 7 mer and $6 \mathrm{mer}$ loci which match with each miRNA seed region [15].

\section{Cell culture}

Human chondrosarcoma cell line SW1353 was purchased from the cell bank of Chinese Academy of Sciences (Shanghai, China). SW1353 cell was cultured in L-15 medium (HyClone, Waltham, MA, USA), in which added 10\% high-quality fetal bovine serum (Invitrogen, Carlsbad, California, USA), 100U/ml penicillin and $100 \mu \mathrm{g} / \mathrm{ml}$ streptomycin (Invitrogen). All cells were cultured in a wet incubator containing $5 \% \mathrm{CO}_{2}$ at $37^{\circ} \mathrm{C}$.

\section{Primer design and vector construction}

Through whole-genome synthesis method, SOX6 complete coding sequence (63 2688bp) was synthesized and constructed into pIRES2-EGFP carrier, SOX6 3'UTR region 1000bp (4300 5300bp) fragment was synthesized and constructed into psi-check-2 carrier and marked as wild type carrier (Wild type). After successful construction of wild type carrier, mutant type carrier (Mutant) was constructed by using point mutation method. All miRNA mimics and inhibitors were synthesized by Genepharma. See Table 1 for sequence:

\section{Cell transfection and luciferase reporter gene assay}

Wild type and mutant type carriers were co-transfected into SW1353 cells together with miRNA mimics and mimic NC in a concentration of 20nM by Lipofectamine 3000 transfection reagents (Invitrogen, Carlsbad, CA, USA), to complete miRNA over expression cotransfection. Wild type and mutant type carriers were co-transfected into SW1353 cells together with miRNA inhibitors and inhibitor NC in a concentration of $20 \mathrm{nM}$ to complete miRNA over expression cotransfection. Cells were lysed after $48 \mathrm{~h}$ of 
culturing, and activities of firefly luciferase and ranilla luciferase in cell lysis buffer were measured by dual-luciferase reporter gene assay kit (E1910) (Promega, Madison, WI, USA).

\section{RNA extraction and real-time polymerase chain reaction (qPCR)}

miRNA mimics, mimic NC, miRNA inhibitors and inhibitor NC were transfected into SW1353 cells by Lipofectamine 3000 transfection reagents with a concentration of $20 \mathrm{nM}$, and then cells were lysed after 48h of culturing; total RNA was extracted by TRIzol reagent (Invitrogen), then transcribed as c DNA by ReverAid (Thermo Scientific). Real-time PCR was conducted by using qPCR SYBR Green Master Mix (High Rox Plus) (Thermo Scientific). qPCR system was 20ul, including 1 ul upstream primer, 1 ul downstream primer, 0.4ul cDNA (1000ng/ul), 10ul Green Master Mix and 7.6ul ultra-pure water. This system was reacted for $60 \mathrm{~s}$ at $95^{\circ} \mathrm{C}$, then $30 \mathrm{~s}$ at $95^{\circ} \mathrm{C} ; 30 \mathrm{~s}$ at annealing temperature and $30 \mathrm{~s}$ at $72^{\circ} \mathrm{C} ; 40$ cycles were done. Finally DNA was detected for $30 \mathrm{~s}$ at $95^{\circ} \mathrm{C}$ and $35 \mathrm{~s}$ at $5^{\circ} \mathrm{C} .2-\Delta \Delta \mathrm{Ct}$ method was used to determine relative gene expression level. See Table 2 for primer sequence:

\section{Western blot analysis (WB)}

After treating the cells with miRNA mimics, miRNA inhibitors, SOX6 overexpression vector and SOX6 SiRNA for $48 \mathrm{~h}$, the cells were collected. $1 \times\left(10^{6}\right)$ cells were cracked by $1 \mathrm{ml}$ of RIPA and $10 \mu \mathrm{l}$ of PMSF, then centrifuged at $12,000 \mathrm{r} / \mathrm{min}$ at $4^{\circ} \mathrm{C}$ for $4 \mathrm{~min}$. The intermediate protein layer solution was removed, and the BCA protein quantification kit was used for protein quantification. Samples of each group were diluted to $50 \mu \mathrm{g} / \mathrm{ml}$, and the diluted protein was mixed with Sample Buffer at the ratio of $4: 1$ and heated at $100^{\circ} \mathrm{C}$ for $5 \mathrm{~min}$. Then Mixing Acrylamide, Resolving Buffer, Starcking Buffer, distilled water, 10\% APS, and TEMED were mixed in proportion to make SDS-PAGE separation gel and stacking gel, and poured into the gel plate. The Prestained Protein Ladder and the sample were separately added into the sample wells of the gel plate, and the proteinloaded SDS-PAGE gel was subjected to vertical gel electrophoresis for $50 \mathrm{~min}$. The polyvinylidene difluoride (PVDF) membrane was activated by methanol for $1 \mathrm{~min}$ and then transmembrane was performed. After that, the PVDF membrane was blocked by $5 \%$ fat-free milk containing TBST solution for $1 \mathrm{~h}$. After blocking, the PVDF membrane was washed by TBST. The WNT antibody (Thermo Fisher Scientific, rabbit polyclonal antibody-Catalog Number PA5-27321) was incubated at $25^{\circ} \mathrm{C}$ for $2 \mathrm{~h}$ at a dilution of $1: 500$, and the second antibody was incubated at $25^{\circ} \mathrm{C}$ for $1 \mathrm{~h}$. Finally, Supersignal West Pico PLUS was used to fill the PVDF membrane and was placed in the iBright FL1000 (Thermo Fisher Scientific) for observation.

\section{Statistical analysis}

The data were presented as mean value $\pm S D$. Statistical difference between groups was determined by two-tailed T test. Statistical difference between groups was analyzed by one-way variance, then StudentNeuman-Keuls multiple comparative test was used to make analysis. All experiments were conducted at least 3 times independently, with similar results; also, typical experiments were shown. It was statistically significant if $P<0.05$. NS $>0.05 \rrbracket^{*} P<0.05 \nabla^{\star \star} P<0.01 \rrbracket^{\star * \star *} P<0.001$. 


\section{Results}

\section{Differently expressed miRNA}

By data analysis, we found 55 differently expressed miRNA in LDH and FJOA, including 21 highly expressed miRNAs in FJOA, 29 lowly expressed miRNAs in FJOA. miRNA details are presented in attached table 1. Expression quantity of miR-338-5p and miR-4776-3p in FJOA was nearly 4 times of that in LDH, which was selected as target miRNA for subsequent experiments (Figure 1A, B).

\section{Prediction of Upstream Transcription Factors of DE-miRNAs.}

We predicted the upstream transcription factors of DE-miRNAs through FunRich software, and the top 10 transcriptions are presented in Figures 2. The top 10 transcription factors of DE-miRNAs were EGR1, SP1, SP4, MEF2A, POU2F1, ZFP161, HOXB9, POU6F1, RORA, SOX6.

\section{Functional annotation and pathway enrichment analysis}

The Enrichr database was utilized to perform GO functional annotation and KEGG pathway enrichment analysis. The GO functional annotation included three categories, namely, biological process (BP), cellular component (CC), and molecular function (MF). The top 10 enriched $\mathrm{GO}$ items are listed in Figure 3. The biological process analysis results show that the biological process of DE-miRNAs is mainly concentrated in regulation of nucleobase, nucleoside, nucleotide and nucleic acid metabolism (Figure 3A), the cellular component is mainly concentrated in nucleus and cytoplasm (Figure 3B), the molecular function is mainly concentrated in transcription factor activity and transcription regulator activity (Figure 3C). The biological process of DE-miRNAs is mainly concentrated in S1P, VEGFP, ErbB signaling network and pathway (Figure 3D).

\section{miRNA target gene prediction}

miR-338-5p and miR-4776-3p target genes were predicted by TargetScanHuman website, which found miR-338-5p binds 4813 genes naturally and miR-4776-3p binds 4175 genes naturally; the details are presented in attached table 2. Various references reported transcription factors SOX5/SOX6 and SOX9 together promoted chondrogenesis in whole genome by super-enhancers [16,17], miR-338-5p and miR4776-3p made target binding with SOX6, so SOX6 was chosen as study target (Figure 4 A, B).

\section{miR-338-5p and miR-4776-3p bind with SOX6}

Through dual-luciferase reporter gene assay, we found exogenous miR-338-5p mimics can bind with 3'UTR of SOX6, allowing its activity to reduce by about $30 \%$, while miR-4776-3p mimics can bind with 3'UTR of SOX6, allowing its activity to reduce by about 30\%. However, it was found SOX6 activity would rise by about $40 \%$ after transfection of miR-338-5p inhibitors and also about $40 \%$ after transfection of miR-4776-3p inhibitors (Figure 5A-D). 
Overexpression of miR-338-5p and miR-4776-3p mimics can increase expression of catabolism, inflammation and apoptosis markers.

By bioinformatics method, it was found expression quantity of miR-338-5p and miR-4776-3p in FJOA was about 4 times of that in control group, and then we further explored how these two miRNAs worked in cartilage degeneration. After miR-338-5p mimics and miR-4776-3p mimics were transfected to SW1353 cells, it was found expression level of catabolism genes (MMP13), inflammation marker gene (TNFA, IL6, and MCP1) and apoptosis marker genes (PARP) was significantly improved. This indicated miR-338-5p and miR-4776-3p can promote catabolism, inflammation and apoptosis, thereby promoting chondrocyte degeneration (Figure 6A, B).

\section{Knock-down of miR-338-5p and miR-4776-3p can inhibit expression of catabolism, inflammation and apoptosis markers}

The experiments showed miR-338-5p and miR-4776-3p mimics enabled to improve catabolism, and trigger inflammation and apoptosis; to further verify whether it was reversed after knock-down of miR338-5p and miR-4776-3p, miR-338-5p and miR-4776-3p inhibitors were transfected to SW1353 cells. qPCR assay found that miR-338-5p and miR-4776-3p inhibitors can inhibit expression of MMP13, TNFA, IL6, MCP1 and PARP. These expressions miR-338-5p and miR-4776-3p played an important role in chondrocyte degeneration (Figure 7A, B).

\section{Impact of miR-338-5p and miR-4776-3p on osteogenesis}

To further verify whether miR-338-5p and miR-4776-3p can affect osteogenesis directly, we conducted over expression and inhibition of miR-338-5p and miR-4776-3p, and found that miR-338-5p and miR4776-3p mimics can inhibit expression of Runx2, ALP, OPN, WNT4 and WNT16 of bone marker genes, and improve expression of osteoclastogenesis marker genes TRACP and CTSK (Figure 8A, B). Yet, miR338-5p and miR-4776-3p inhibitors can improve expression of Runx2, ALP, OPN, WNT4 and WNT16, and inhibit expression of TRACP and CTSK (Figure 8C, D). WB results showed that miR-338-5p and miR-4776$3 p$ overexpression can inhibit expression of WNT4 and miR-338-5p and miR-4776-3p knockdown can improve expression of WNT4 at the protein level (Figure 8E). This indicated miR-338-5p and miR-4776-3p can directly affect osteogenesis.

\section{SOX6 mediates the expression of osteogenic genes and osteoclast genes}

To verify whether the changes in the expression levels of osteogenic and osteoclast markers upon overexpression or inhibition of miR-338-5p and miR-4776-3p are directly caused by changes in SOX6, we overexpressed and knockdown SOX6 in human SW1353 cells. By qRT-PCR, we found that the expression of Runx2, ALP, OPN, WNT4 and WNT16 were significantly upregulated and the expression of TRACP and CTSK was significantly downregulated when SOX6 overexpression (Fig. 9A). The expression of RUnX2, ALP, OPN, WNT4 and WNT16 were significantly downregulated and the expression of TRACP and CTSK was significantly upregulated when SOX6 knockdown (Fig. 9B). Overexpression of miR-338-5p and 
miR-4776-3p can reduce the expression of WNT4 at the protein level, while knockdown of miR-338-5p and miR-4776-3p can increase the expression of WNT4 (Fig. 9C).

\section{Discussion}

The most challenging aspect in the treatment of articular cartilage injury mainly includes determination of matrix changes and cells and molecular mechanism of chondrocyte differentiation and of dedifferentiation behavior, and understanding of how to affect structural integrity and tissue remodeling of articular cartilage [18]. Several studies have showed that miRNA in tissues played a vital role in cellular processes (such as apoptosis, cell proliferation, differentiation, metabolism and cell cycle control) [19]. Several existing studies have shown that miRNA may be related to premature degradation of cartilage $[14,20]$, and some studies also have indicated multiple miRNA had higher expression quantity in cartilage with arthritis [21-23]. However, most studies involved compassion of miRNA expression quantity between samples of osteoarticular cartilage and normal samples, and did not go into the molecular mechanism of cartilage degeneration caused by miRNA. In this paper, we preliminarily studied the molecular mechanism of cartilage degeneration caused by miR-338-5p and miR-4776-3p, providing new ideas for further studying cartilage injury and degeneration.

Throughout the study, we intended to find miRNA with significantly high expression of cartilage in osteoarthritis, and study the role of miRNA in cartilage lesions. We downloaded 2100 miRNAs expression data of LDH patients and FJOA patients from NCBI GEO database, and made bioinformatics analysis, which found miR-4776-3p and miR-338-5p showed significantly high expression in FJOA. To determine miR-338-5p and miR-4776-3p have physiological and pathological actions action in articular cartilage degeneration or not, miR-338-5p and miR-4776-3p were over expressed and knocked down in chondrocyte SW1353, so as to determine their impact on cartilage. Through detection of expression quantity of catabolism, inflammatory reaction and apoptosis marker genes, we found miR-338-5p and miR-4776-3p can improve catabolism and trigger apoptosis, thereby promoting cartilage degeneration. Through dualluciferase reporter gene assay, miR-338-5p and miR-4776-3p can bind with cartilage-related gene SOX6 naturally, but further study on the molecular mechanism of cartilage lesion and the role of SOX6 in in vivo is required.

Related studies have shown that cartilage in osteoarthritis usually accompanies bone lesions. Joint integrity comes from balance of physiological interaction between skeletons and cartilages. Accumulated evidences in the bodies also indicated the increase of bone absorption occurred at early stage of osteoarthritis progression, and cytokines blocking bone absorption can prevent cartilage injury, thereby confirming the role of bone factors in crosstalk of the two tissues [24]. In this study, we also preliminarily studied the role of miR-338-5p and miR-4776-3p in bone formation and absorption, which found they can inhibit osteogenesis and promote osteoclastogenesis. However, we did not study correlation and molecular mechanism of miR-338-5p and miR-4776-3 between bone formation and absorption in cartilage lesions, which will be the focus in future studies. 


\section{Conclusions}

To sum up, we, by using bioinformatics methods in the whole study, found miR-338-5p and miR-4776-3p had significantly different high expression quantity of cartilage in osteoarthritis, and verified miR-338-5p and miR-4776-3p can improve catabolism and trigger inflammation and apoptosis by experiments. At the same time, they can promote osteoclastogenesis and inhibit osteogenesis. These study results showed that miR-338-5p and miR-4776-3p played an important role in cartilage lesion, and can be used as diagnostic marks of early cartilage lesions.

\section{Declarations}

\section{Authors' contributions}

Wang $\mathrm{J}$ and Guan $\mathrm{X}$ analyzed the data and drafted the manuscript. Zhang $\mathrm{L}$, Shao S, Gao B, Li H, Huo L, Huang $\mathrm{S}$ done the experiments and preformed statistical analysis of the data. Wu $\mathrm{J}$ and Cui $\mathrm{X}$ revised the manuscript. All authors read and approved the final manuscript.

\section{Funding}

This research was funded by Strategic Support Force Medical Center Foundation (19ZX10), China.

\section{Availability of data and materials}

All data generated or analyzed during this study are included in this published article.

\section{Ethics approval and consent to participate}

Not applicable.

\section{Consent for publication}

Not applicable.

\section{Competing interests}

The authors declare that they have no competing interests.

\section{References}

1. Glyn-Jones S, Palmer AJ, Agricola R, et al. Osteoarthritis Lancet. 2015;386(9991):376-87. https://doi.org/10.1016/S0140-6736(14)60802-3.

2. Sacitharan PK. Ageing and osteoarthritis. Sub-cellular Biochem. 2019;91:123-59. https://doi.org/10.1007/978-981-13-3681-2_6. 
3. Pitsillides AA, Beierm F. Cartilage biology in osteoarthritis-lessons from developmental biology. Nat Rev Rheumatol. 2011;7(11):654-63. https://doi.org/10.1038/nrrheum.2011.129.

4. Ambros V. The functions of animal microRNAs. Nature. 2004;431:350-5. https://doi.org/10.1038/nature02871.

5. Ha M, Kim VN. Regulation of microRNA biogenesis. Nat Rev Mol Cell Biol. 2014;15(8):509-24. https://doi.org/10.1038/nrm3838.

6. Tang P, Xiong Q, Ge W, et al. The role of microRNAs in osteoclasts and osteoporosis. RNA Bio. 2014;11(11):1355-63. https://doi.org/10.1080/15476286.2014.996462.

7. Guay C, Regazzi R. Circulating microRNAs as novel biomarkers for diabetes mellitus. Nat Rev Endocrinol. 2013;9(9):513-21. https://doi.org/10.1038/nrendo.2013.86.

8. Ji C, Guo X. The clinical potential of circulating microRNAs in obesity. Nat Rev Endocrinol. 2019;15:731-43. https://doi.org/10.1038/s41574-019-0260-0.

9. Lin S, Gregory RI. MicroRNA biogenesis pathways in cancer. Nat Rev Cancer. 2015;15(6):321-33. https://doi.org/10.1038/nrc3932.

10. Yue J, Song D, Lu W, et al. Expression profiles of inflammation-associated microRNAs in periapical lesions and human periodontal ligament fibroblasts inflammation. J Endodont. 2016;42(12):1773-8. https://doi.org/10.1016/j.joen.2016.08.013.

11. Meng F, He A, Zhang Z, et al. Chondrogenic differentiation of ATDC5 and hMSCs could be induced by a novel scaffold-tricalcium phosphate-collagen-hyaluronan without any exogenous growth factors in vitro. J Biomed Mater Res A. 2014;102(8):2725-35. https://doi.org/10.1002/jbm.a.34948.

12. Li Y, Zhu H, Zeng $X$, et al. Suppression of autophagy enhanced growth inhibition and apoptosis of interferon- $\beta$ in human glioma cells. Mol Neurobiol. 2013;47(3):1000-10. https://doi.org/10.1007/s12035-013-8403-0.

13. Livak KJ, Schmittgen TD. Analysis of relative gene expression data using real-time quantitative PCR and the 2(-Delta Delta C(T)) method. Methods. 2001;25(4):402-8. https://doi.org/10.1006/meth.2001.1262.

14. Zhang Y, Lin J, Zhou X, et al. Melatonin prevents osteoarthritis-induced cartilage degradation via targeting MicroRNA-140. Oxid Med Cell Longe. 2019;2019:9705929. https://doi.org/10.1155/2019/9705929.

15. Lewis BP, Burge CB, Bartel DP. Conserved seed pairing, often flanked by adenosines, indicates that thousands of human genes are microRNA targets. Cell. 2005;120(1):15-20. https://doi.org/10.1016/j.cell.2004.12.035.

16. Nishimura R, Hata K, Takahata Y, et al. Regulation of cartilage development and diseases by transcription factors. J Bone Metab. 2017;24(3):147-53. https://doi.org/10.11005/jbm.2017.24.3.147.

17. Liu CF, Lefebvre V. The transcription factors SOX9 and SOX5/SOX6 cooperate genome-wide through super-enhancers to drive chondrogenesis. Nucleic Acids Res. 2015;43(17):8183-203. https://doi.org/10.1093/nar/gkv688. 
18. Carballo CB, Nakagawa Y, Sekiya I, et al. Basic science of articular cartilage. Clin Sports Med. 2017;36(3):413-25. https://doi.org/10.1016/j.csm.2017.02.001.

19. Cortez MA, Welsh JW, Calin GA. Circulating microRNAs as noninvasive biomarkers in breast cancer. Recent Res Cancer Res. 2012;195:151-61. https://doi.org/10.1007/978-3-642-28160-0_13.

20. Tao SC, Yuan T, Zhang YL, et al. Exosomes derived from miR-140-5p-overexpressing human synovial mesenchymal stem cells enhance cartilage tissue regeneration and prevent osteoarthritis of the knee in a rat model. Theranostics. 2017;7(1):180-95. https://doi.org/10.7150/thno.17133.

21. Gibson G, Asahara H. microRNAs and cartilage. J Orth Res. 2013;31(9):1333-44. https://doi.org/10.1002/jor.22397.

22. Skrzypa M, Szala D, Gablo N, et al. miRNA-146a-5p is upregulated in serum and cartilage samples of patients with osteoarthritis. Polski Przeglad Chirurgiczny. 2019;91(3):1-5. https://doi.org/10.5604/01.3001.0013.0135.

23. Ntoumou E, Tzetis M, Braoudaki M, et al. Serum microRNA array analysis identifies miR-140-3p, miR$33 b-3 p$ and miR-671-3p as potential osteoarthritis biomarkers involved in metabolic processes. Clin Epigen. 2017;9:127. https://doi.org/10.1186/s13148-017-0428-1.

24. Funck-Brentano T, Cohen-Solal M. Crosstalk between cartilage and bone: when bone cytokines matter. Cyto Growth Factor Rev. 2011;22(2):91-7. https://doi.org/10.1016/j.cytogfr.2011.04.003.

\section{Tables}

Table 1. Oligonucleotide sequences synthesized in the present study

\begin{tabular}{|ll|}
\hline Name & Sequences $\left(\mathbf{5}^{\prime} \mathbf{3}^{\prime}\right.$ ') \\
\hline miR-338-5p mimics-F & AACAAUAUCCUGGUGCUGAGUG \\
\hline miR-338-5p mimics-R & CUCAGCACCAGGAUAUUGUUUU \\
\hline miR-4776-3p mimics-F & CUUGCCAUCCUGGUCCACUGCAU \\
\hline miR-4776-3p mimics-R & GCAGUGGACCAGGAUGGCAAGUU \\
\hline miR-338-5p inhibitors & CACUCAGCACCAGGAUAUUGUU \\
\hline miR-4776-3p inhibitors & AUGCAGUGGACCAGGAUGGCAAG \\
\hline NC mimics & UUCUUCGAACGUGUCACGUTT \\
\hline NC inhibitors & CAGUACUUUUGUGUAGUACAA \\
\hline
\end{tabular}

Table 2. PCR primer sequences in the present study 


\begin{tabular}{|c|c|c|}
\hline Name & Sequences $\left(5^{\prime}-3^{\prime}\right)$ & Temperature \\
\hline hsa- $\beta$-actin-F & GAGAAAATCTGGCACCACACCT & - \\
\hline hsa- $\beta$-actin-R & GCACAGCCTGGATGCAACGTA & - \\
\hline has-MMP13-F & ACTGAGAGGCTCCGAGAAATG & $63^{\circ} \mathrm{C}$ \\
\hline has-MMP13-R & GAACCCCGCATCTTGGCTT & $63^{\circ} \mathrm{C}$ \\
\hline has-TNFA-F & ССТСТСТСТААTСАGСССТСТG & $60^{\circ} \mathrm{C}$ \\
\hline has-TNFA-R & GAGGACCTGGGAGTAGATGAG & $60^{\circ} \mathrm{C}$ \\
\hline has-IL-6-F & АСTСАССТСTTCAGAACGAATTG & $63^{\circ} \mathrm{C}$ \\
\hline has-IL-6-R & CCATCTTTGGAAGGTTCAGGTTG & $63^{\circ} \mathrm{C}$ \\
\hline has-MCP1-F & CAGCCAGATGCAATCAATGCC & $60^{\circ} \mathrm{C}$ \\
\hline has- MCP1-R & TGGAATCCTGAACCCACTTCT & $60^{\circ} \mathrm{C}$ \\
\hline has-PARP-F & CGGAGTCTTCGGATAAGCTCT & $62^{\circ} \mathrm{C}$ \\
\hline has- PARP-R & TTTCCATCAAACATGGGCGAC & $62^{\circ} \mathrm{C}$ \\
\hline hsa-Runx $2-\mathrm{F}$ & ТСТТСАСАААТССТСССС & $52^{\circ} \mathrm{C}$ \\
\hline hsa-Runx2-R & TGGATTAAAAGGACTTGG & $52^{\circ} \mathrm{C}$ \\
\hline hsa- $A / p-\mathrm{F}$ & GACAAGAAGCCCTTCACTGC & $59^{\circ} \mathrm{C}$ \\
\hline hsa-Alp-R & AGACTGCGCCTGGTAGTTGT & $59^{\circ} \mathrm{C}$ \\
\hline hsa-OPN-F & ACTCGAACGACTCTGATGATGT & $57^{\circ} \mathrm{C}$ \\
\hline hsa-OPN-R & GTCAGGTCTGCGAAACTTCTTA & $57^{\circ} \mathrm{C}$ \\
\hline hsa-WNT4-F & AGGAGGAGACGTGCGAGAAA & $60^{\circ} \mathrm{C}$ \\
\hline hsa-WNT4-R & CGAGTCCATGACTTCCAGGT & $60^{\circ} \mathrm{C}$ \\
\hline hsa-WNT16-F & TTCAGACACGAGAGATGGAACT & $63^{\circ} \mathrm{C}$ \\
\hline hsa-WNT16-R & CCAGCCTTCACTTGCTGAG & $63^{\circ} \mathrm{C}$ \\
\hline hsa-TRACP-F & GACTGTGCAGATCCTGGGTG & $63^{\circ} \mathrm{C}$ \\
\hline hsa-TRACP-R & GGTCAGAGAATACGTCCTAAAAG & $63^{\circ} \mathrm{C}$ \\
\hline hsa-CTSK-F & ACTCAAAGTACCСCTGTCTCAT & $62^{\circ} \mathrm{C}$ \\
\hline hsa-CTSK-R & CCACAGAGCTAAAAGCCCAAC & $62^{\circ} \mathrm{C}$ \\
\hline
\end{tabular}


A

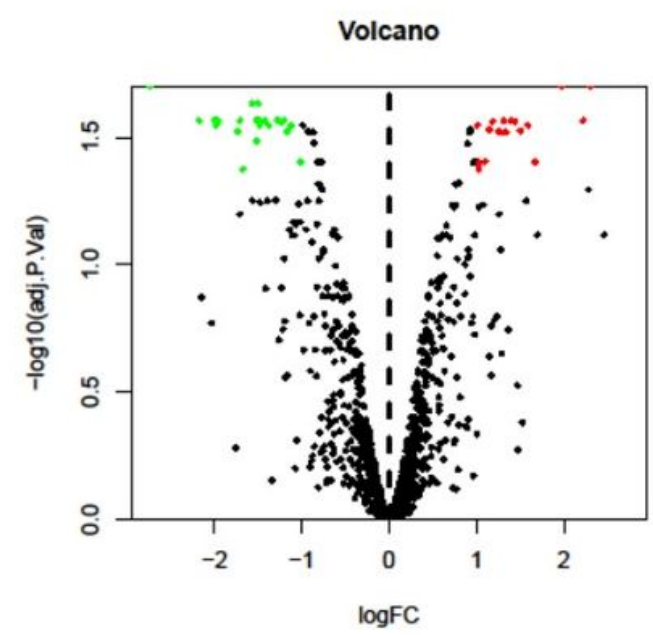

B

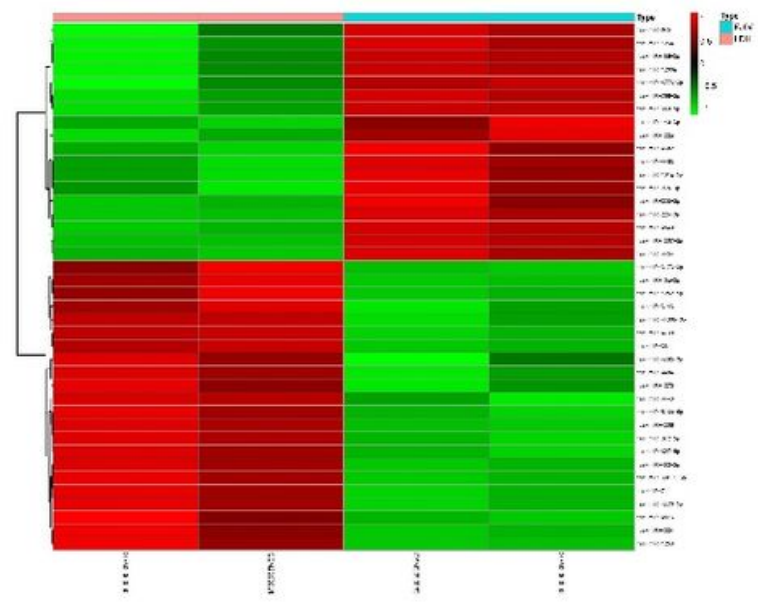

\section{Figure 1}

Differently expressed miRNA. (A) Volcano plot of miRNA expression. (B) Differently expressed miRNA heat map.

\section{TFs for differential miRNA}

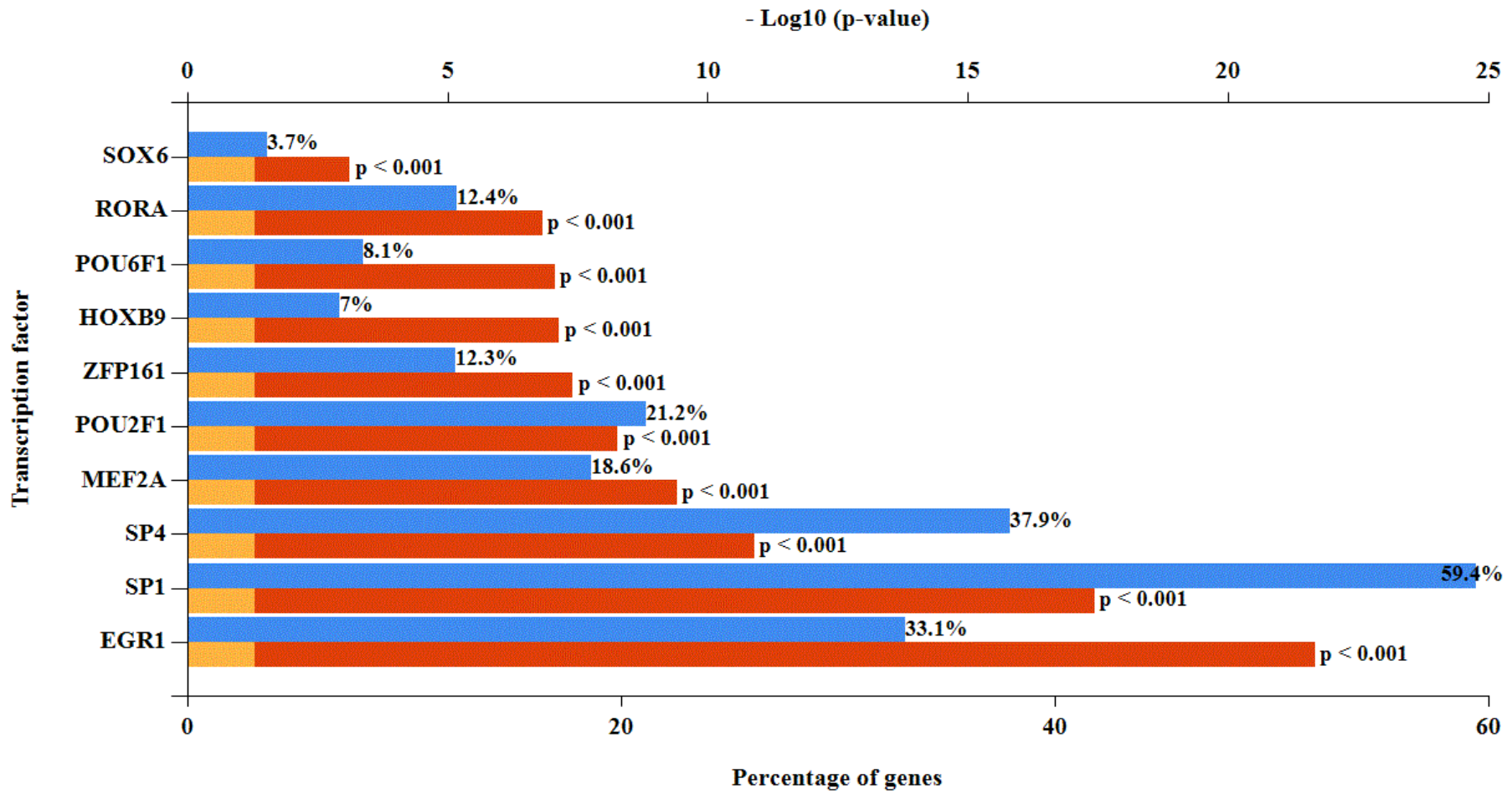

Figure 2 
The top ten upstream transcription factors of upregulated DE-miRNAs.

A

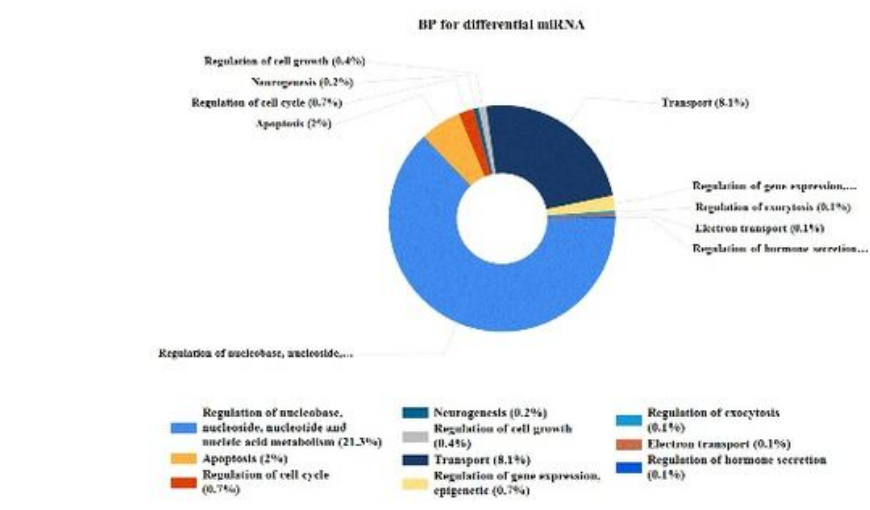

C

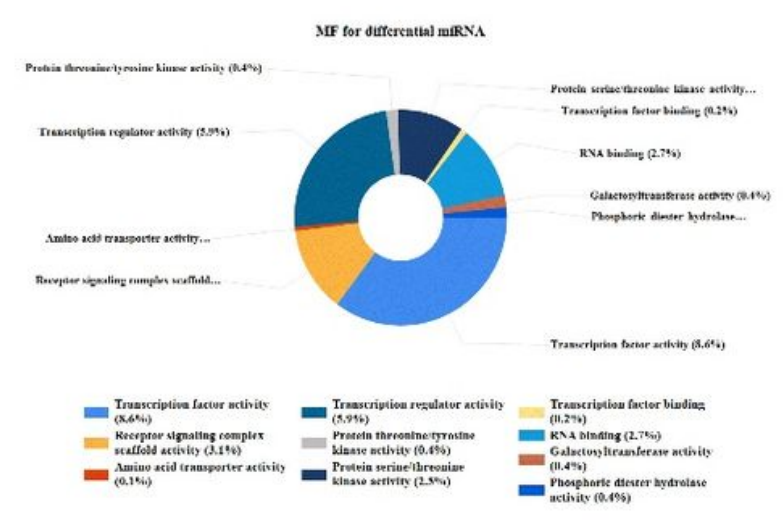

B

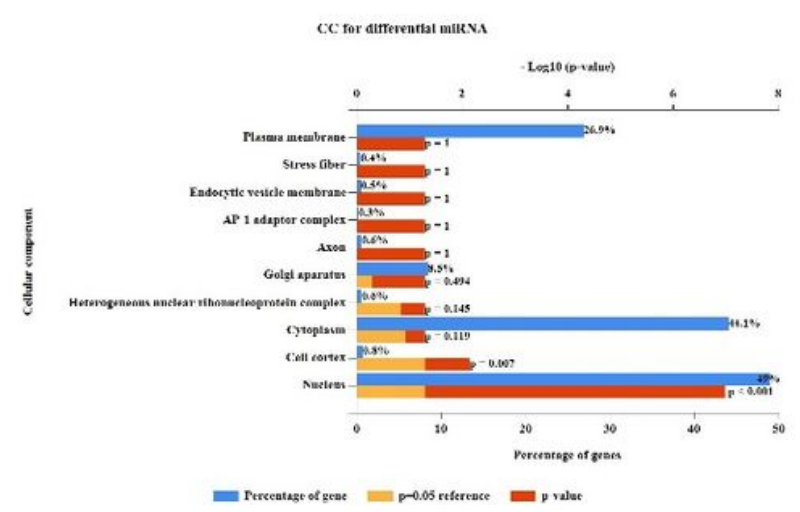

D

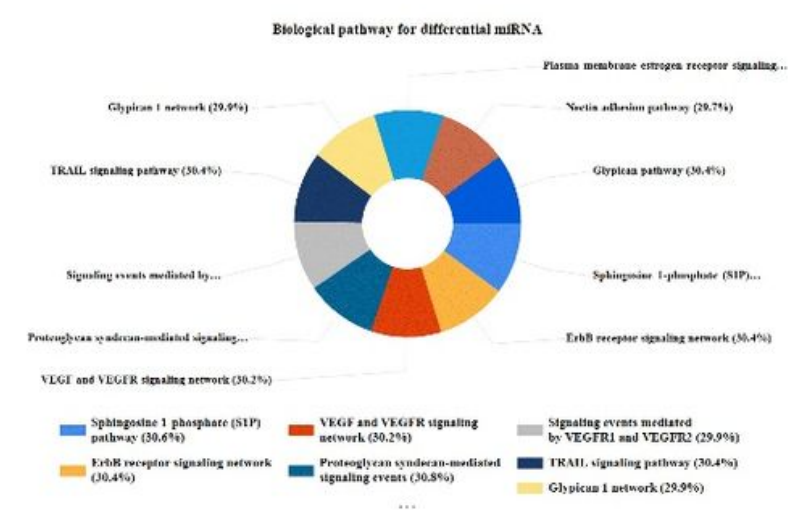

Figure 3

GO functional annotation and KEGG pathways of DE-miRNAs. (A) The top 10 enriched BP items of DEmiRNA. (B) The top 10 enriched CC items of DE-miRNA. (C) The top 10 enriched MF items of DE-miRNA candidate target genes. (D) The top 10 enriched KEGG pathways of DE-miRNA.

A

Position 4604-4611 of SOX6 3' UTR $\quad$ 5' ...UCUCAAAGUAUAAUAAUAUUGUA...

hsa-miR-338-5p

3' GUGAGUCGUGGUCCUAUAACAA

B

Position 1556-1562 of SOX6 3' UTR $\quad 5 ' \quad$...UCAGAGCAAAGAUGGUGGCAAAU...

hsa-miR-4776-3p

3' UACGUCACCUGGUCCUACCGUUC 
Figure 4

miR-338-5p and miR-4776-3p bind with S0X6 naturally. (A) miR-338-5p bind with SOX6 naturally. (B) miR4776-3p bind with SOX6 naturally.

A

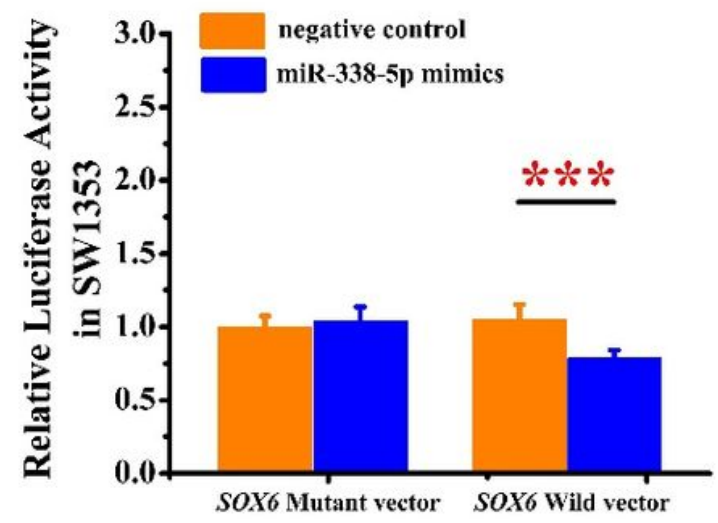

C

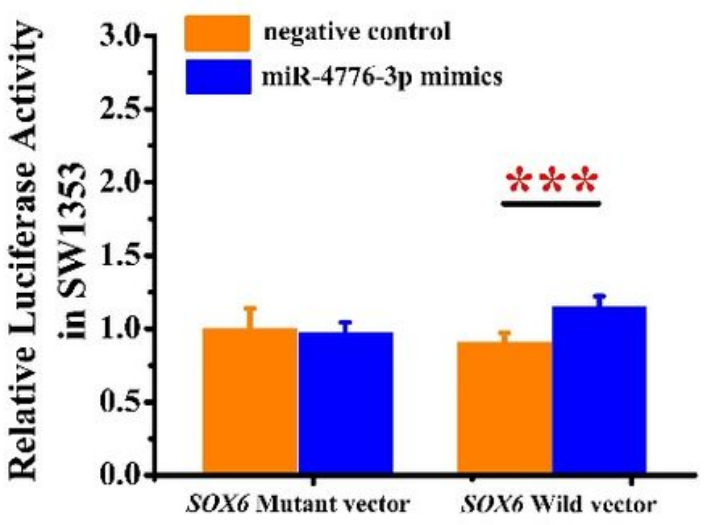

\section{B}

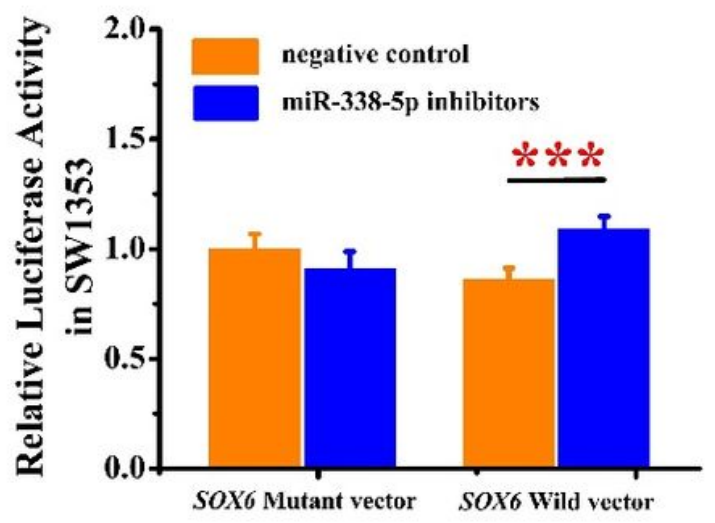

D

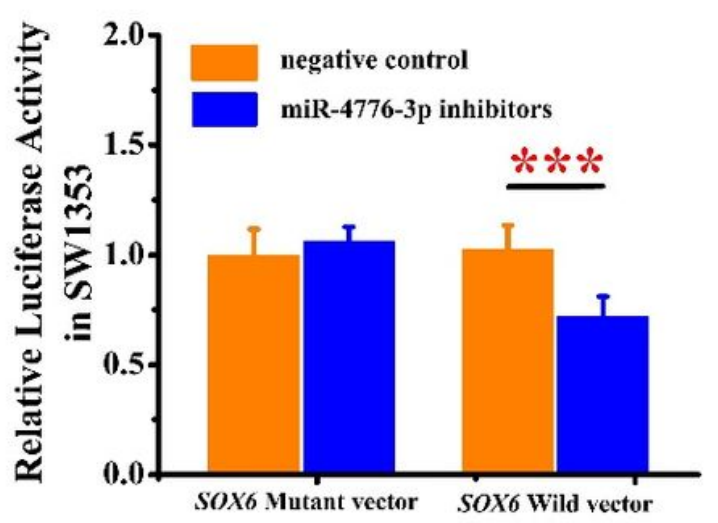

\section{Figure 5}

miR-338-5p and miR-4776-3p bind with SOX6 and inhibit SOX6 expression. (A) miR-338-5p mimics bind with SOX6 and inhibit SOX6 expression. (B) miR-338-5p inhibitors improve SOX6 expression. (C) miR4776-3p mimics bind with SOX6 and inhibit SOX6 expression. (D) miR-4776-3p inhibitors improve SOX6 expression. 

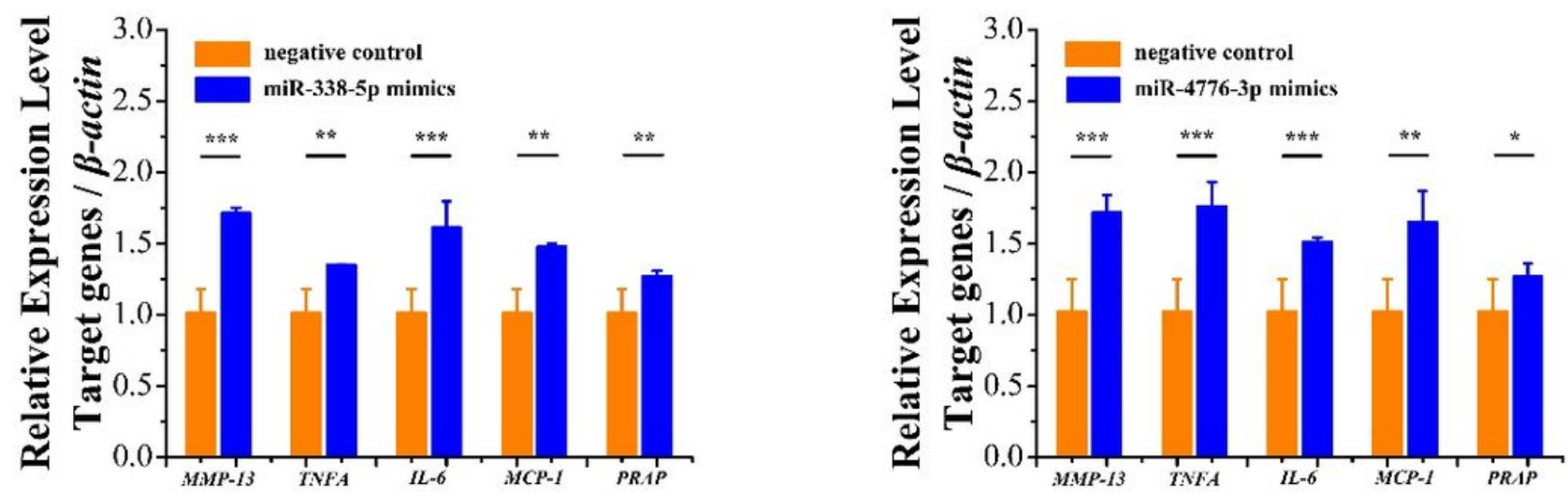

Figure 6

Over expressed miR-338-5p and miR-4776-3p improve catabolism, and trigger inflammation and apoptosis. (A) miR-338-5p mimics improve gene expression of MMP13, TNFA, IL6, MCP1 and PARP. (B) miR-4776-3p mimics improve gene expression of MMP13, TNFA, IL6, MCP1 and PARP.

A

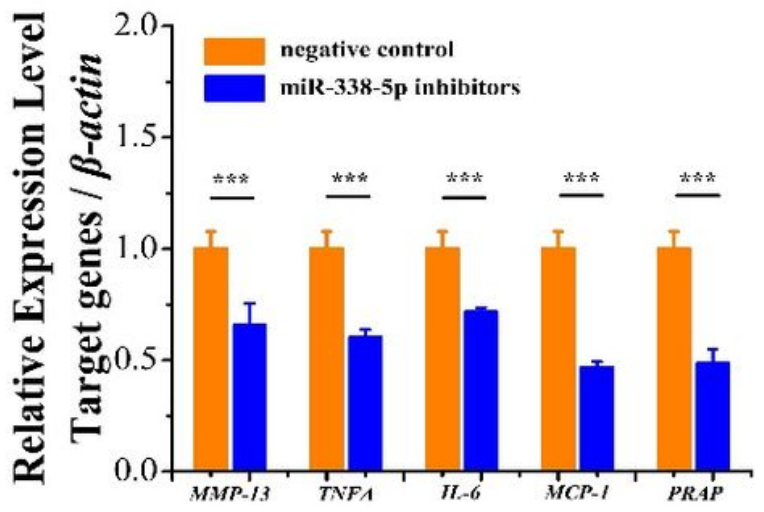

B

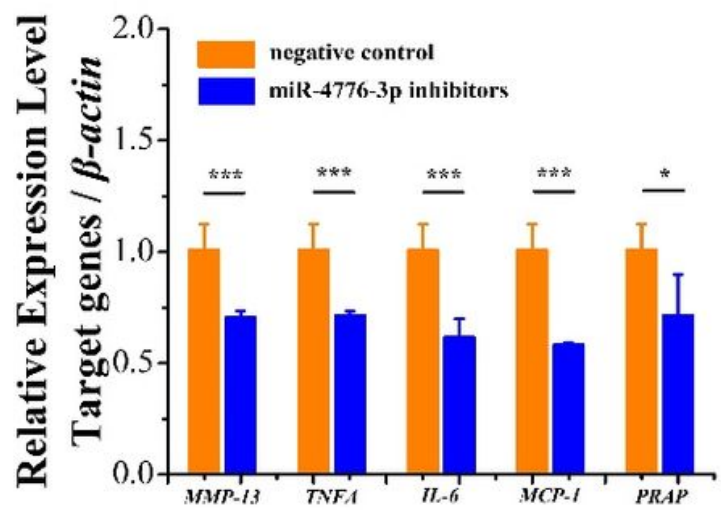

Figure 7

Knock-down of miR-338-5p and miR-4776-3p inhibit catabolism, and trigger inflammation and apoptosis. (A) miR-338-5p inhibitors reduce gene expression of MMP13, TNFA, IL6, MCP1 and PARP. (B) miR-4776$3 p$ inhibitors reduce gene expression of MMP13, TNFA, IL6, MCP1 and PARP. 

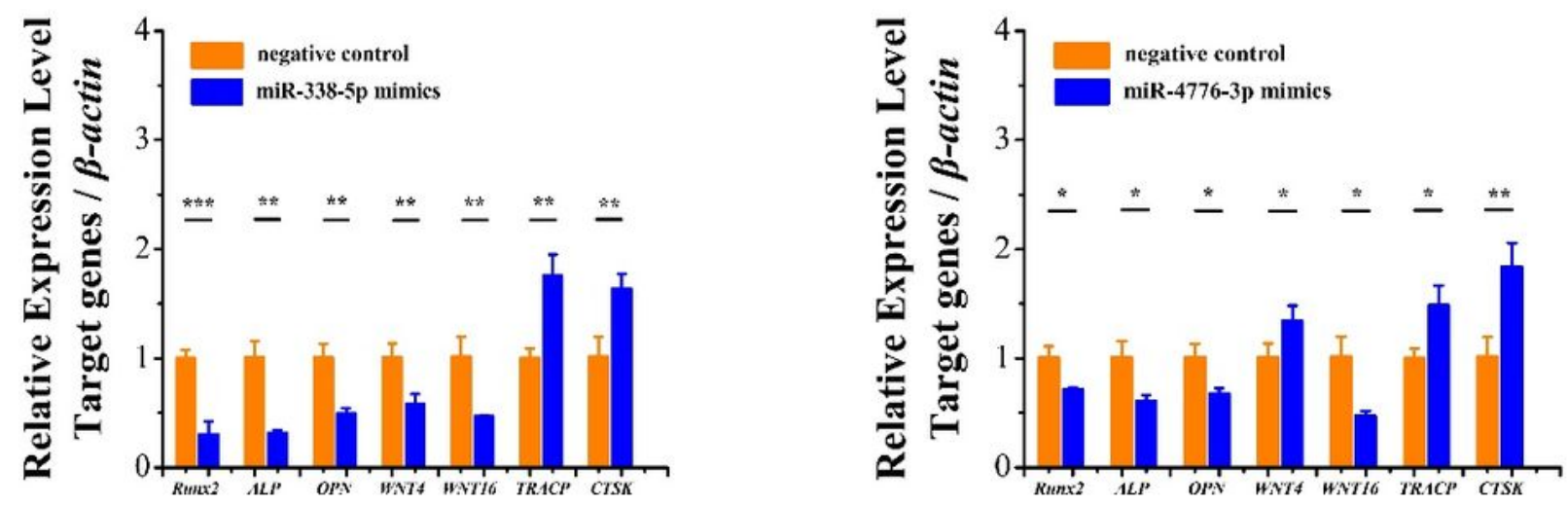

C

D
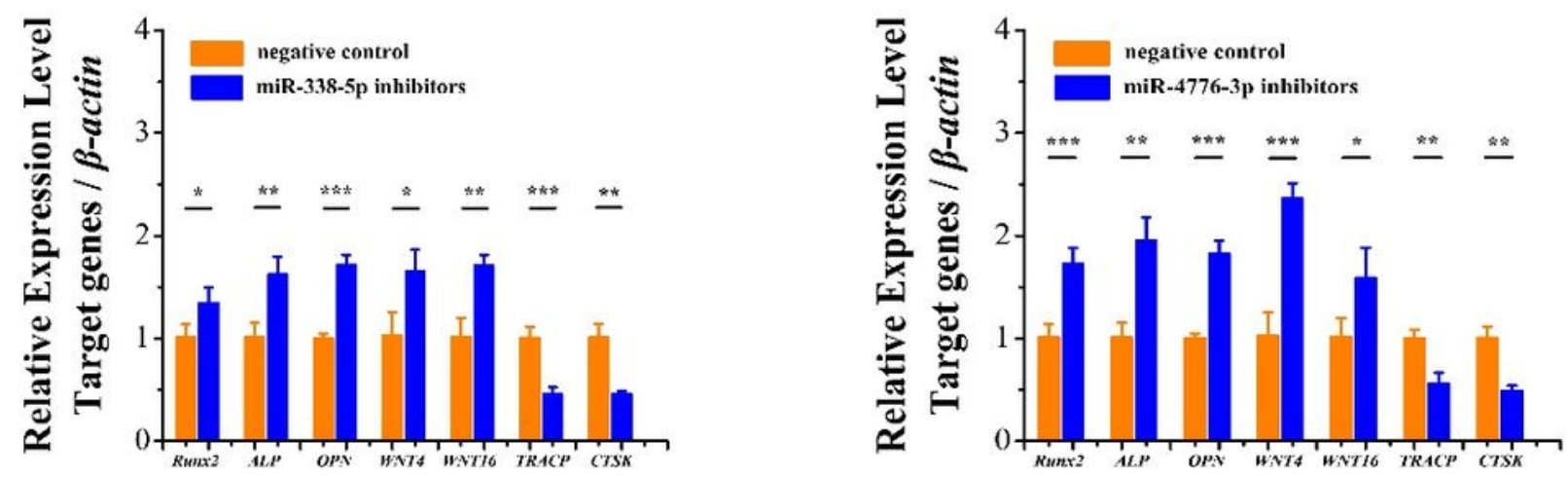

$\mathbf{E}$

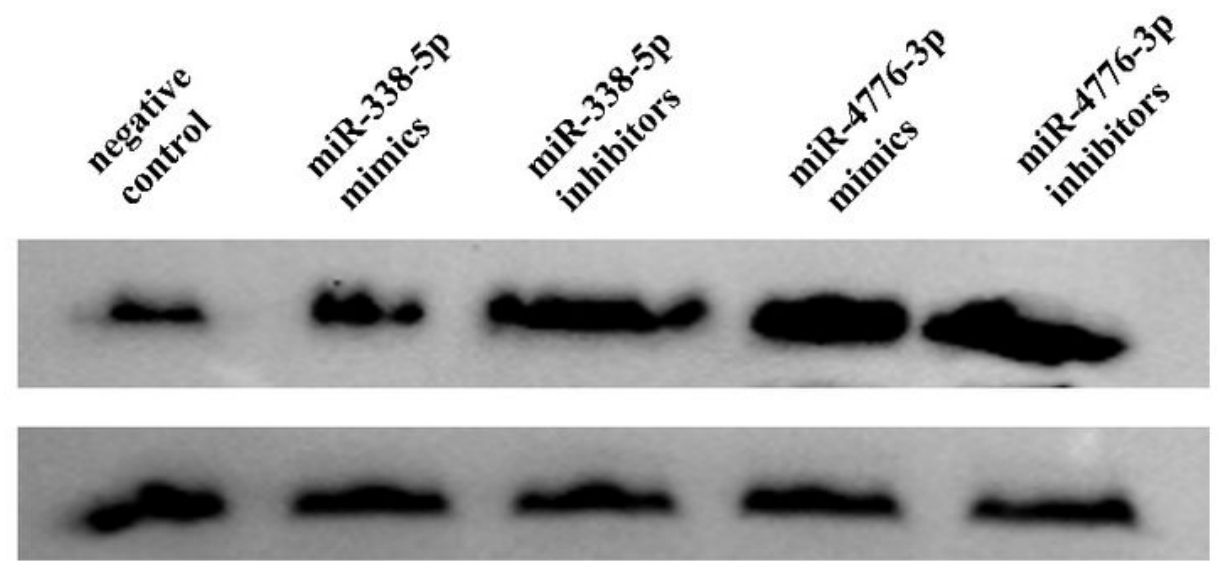

WNT4

$\beta$-actin

\section{Figure 8}

miR-338-5p and miR-4776-3p affect osteogenesis. (A) miR-338-5p mimics inhibit gene expression of Runx2, ALP, OPN, WNT4 and WNT16, and promote expression of osteoclastogenesis genes TRACP and CTSK. (B) miR-4776-3p mimics inhibit gene expression of Runx2, ALP, OPN, WNT4 and WNT16, and promote expression of osteoclastogenesis genes TRACP and CTSK. (C) miR-338-5p inhibitors promote gene expression of Runx2, ALP, OPN, WNT4 and WNT16, and inhibit expression of osteoclastogenesis 
genes TRACP and CTSK. (D) miR-4776-3p inhibitors promote gene expression of Runx2, ALP, OPN, WNT4 and WNT16, and inhibit expression of osteoclastogenesis genes TRACP and CTSK. (E) miR-338-5p mimics and miR-4776-3p mimics inhibit the expression of WNT4, miR-338-5p inhibitors and miR-4776-3p inhibitors increased the expression of WNT4 at the protein level.

A

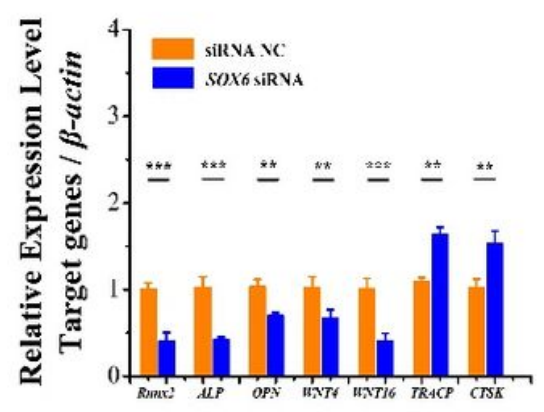

B

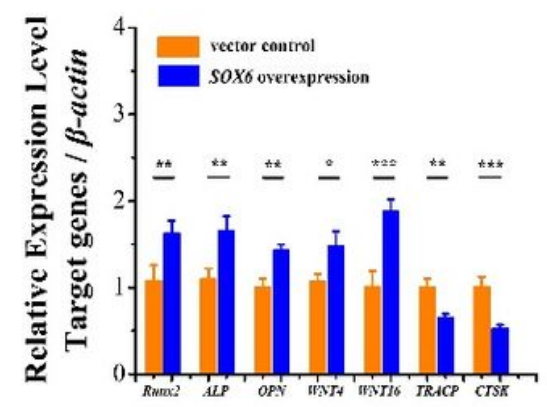

C

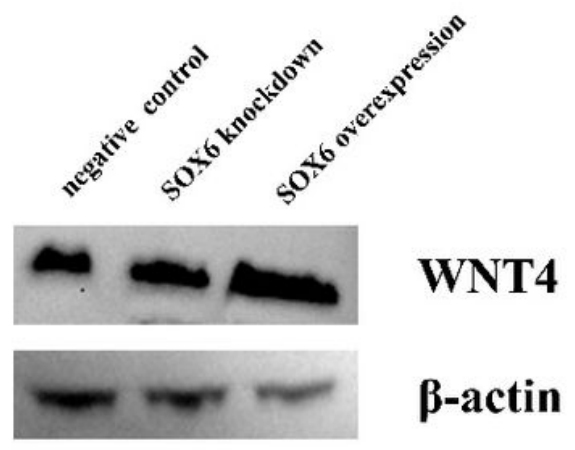

\section{Figure 9}

SOX6 mediates the expression of osteogenic genes and osteoclast genes. (A) Knockdown of SOX6 significantly suppressed the expression the expression of Runx2, ALP, OPN, WNT4, WNT16 and increased the expression of TRACP and CTSK. (B) Overexpression of SOX6 significantly increased the expression of Runx2, ALP, OPN, WNT4, WNT16 and suppressed the expression of TRACP and CTSK. (C) Knockdown of miR-338-5p and miR-4776-3p can increase the expression of WNT4, while overexpression of miR-338-5p and miR-4776-3p can reduce the expression of WNT4 at the protein level (Fig. 9C). 